This item was submitted to Loughborough's Research Repository by the author.

Items in Figshare are protected by copyright, with all rights reserved, unless otherwise indicated.

\title{
Modelling suppressed muscle activation by means of an exponential sigmoid function: validation and bounds
}

PLEASE CITE THE PUBLISHED VERSION

http://dx.doi.org/10.1016/j.jbiomech.2015.01.009

\section{PUBLISHER}

(c) Elsevier Ltd.

\section{VERSION}

AM (Accepted Manuscript)

\section{PUBLISHER STATEMENT}

This work is made available according to the conditions of the Creative Commons Attribution-NonCommercialNoDerivatives 4.0 International (CC BY-NC-ND 4.0) licence. Full details of this licence are available at: https://creativecommons.org/licenses/by-nc-nd/4.0/

\section{LICENCE}

CC BY-NC-ND 4.0

\section{REPOSITORY RECORD}

Voukelatos, Dimitrios, and Matthew T.G. Pain. 2019. "Modelling Suppressed Muscle Activation by Means of an Exponential Sigmoid Function: Validation and Bounds". figshare. https://hdl.handle.net/2134/17534. 


\title{
Modelling suppressed muscle activation by means of an exponential sigmoid function: Validation and bounds.
}

\author{
Dimitrios Voukelatos and Matthew TG Pain
}

School of Sport, Exercise and Health Sciences, Loughborough University, UK.

\begin{abstract}
The aim of this study was to establish how well a three-parameter sigmoid exponential function, DIFACT, follows experimentally obtained voluntary neural activation-angular velocity profiles and how robust it is to perturbed levels of maximal activation. Six male volunteers (age $26.3 \pm 2.73$ years) were tested before and after an 8-session, 3-week training protocol. Torque-angular velocity $(\mathrm{T}-\omega)$ and experimental voluntary neural drive-angular velocity (\%VA$\omega)$ datasets, obtained via the interpolated twitch technique, were determined from pre- and post-training testing sessions. Non-linear regression fits of the product of DIFACT and a Hill type tetanic toque function and of the DIFACT function only were performed on the pre- and post-training T- $\omega$ and \%VA- $\omega$ datasets for three different values of the DIFACT upper bound, $\alpha_{\max }, 100 \%, 95 \% \& 90 \%$. The determination coefficients, $\mathrm{R}^{2}$, and the RMS of the fits were compared using a two way mixed ANOVA and results showed that there was no significant difference $(p<0.05)$ due to changing $\alpha_{\max }$ values indicating the DIFACT remains robust to changes in maximal activation. Mean $\mathrm{R}^{2}$ values of 0.95 and 0.96 for pre- and post-training sessions show that the maximal voluntary torque function successfully reproduces the $T-\omega$ raw dataset.
\end{abstract}

Key words: interpolate twitch, force, velocity, quadriceps

\section{INTRODUCTION}

In vivo measurements of the maximum voluntary force-velocity relationship show differences to the in vitro tetanic profile, with eccentric forces not increasing much above isometric and tending to decline with increasing lengthening velocity (Westing, 1988; Dudley et al., 1990; Weber \& Kriellaars, 1997). This difference could be due to a neural, tension-limiting mechanism that reduces maximal neural drive at high levels of muscular tension (Westing et al., 1991; Pain \& Forrester, 2009; Pain et al., 2013). Yeadon et al. (2006) represented the in vivo maximum voluntary torquevelocity relationship as a product of a theoretical four parameter Hill-type tetanic torque function, and a three parameter differential activation function (DIFACT). The latter representing the net reduction in neural drive to the muscle with low neural activation at high eccentric velocities to full activation at high concentric velocities. However, the DIFACT function was not explicitly based on measured neural changes and its validity was implicitly assumed through the ability of the combined seven parameter function to reproduce the in vivo torque-velocity profiles. Furthermore, due to its quadratic form, the DIFACT function had multiple equivalent solutions and is difficult to manipulate algebraically. Pain \& Forrester (2009) used a sigmoid exponential function to represent the DIFACT function in order to simplify mathematical manipulation when finding solutions for the seven parameter MVC torque function (MVC). Again the function was only implicitly shown to be successful through scaling of voluntary EMG signals (Pain \& Forrester, 2009). 
Therefore, although now used repeatedly (Lewis et al. 2012; Forrester et al., 2011; Tillin et al., 2012; Pain et al., 2013) in the literature the DIFACT function has yet to be verified in an explicit way. The aims of this study were (i) to establish experimentally how well the DIFACT function follows the in vivo voluntary neural activation-angular velocity profiles in a group of subjects; and (ii) to test the robustness of the exponential DIFACT function to perturbed upper levels of maximal activation.

\section{METHODS}

Measurements from six male volunteers (age $26.3 \pm 2.7$ years, body mass $72.9 \pm$ $11.7 \mathrm{~kg}$, height, $172.2 \pm 8.4 \mathrm{~cm}$; mean \pm SD) tested before and after eight sessions (over three weeks) of high velocity strength training on an isovelocity dynamometer were used as the raw data to address the aims of this study. They all gave written, informed consent and the study was conducted in accordance with the approval given by Loughborough University Ethical Advisory Committee. In brief, testing followed similar methods to those outlined in Yeadon et al. (2006) for the data collection (maximal isometric trials at five knee angles spanning the range of motion and maximal isovelocity trials at 50,100, 150, $250 \& 350 \%$ s) and Forrester et al. (2011) for the data processing and fitting procedures to determine the seven parameter function to describe the in vivo torque-velocity profiles. However, there was the addition of a repeat set of measures at all test velocities where supramaximal electrical stimulation of the femoral nerve was carried out using doublet stimulation (Folland \& Williams, 2007). The interpolated twitch technique (ITT) was used with these stimulated data to determine the percentage of maximal voluntary neural drive (\%VA) during each concentric and eccentric velocity at the optimum joint angle for torque production.

$$
\% \mathrm{VA}=\left(1-\frac{\text { superimposed twitch }}{\text { control twitch evoked at rest }}\right) \times 100
$$

This resulted in: measured torque-velocity data, level of neural drive, and the determination of the three parameters associated with the DIFACT function. In order to establish: a) how well the DIFACT function follows the in vivo voluntary neural activation-angular velocity profiles and $b$ ) whether different values of the the DIFACT upper bound, $\alpha_{\max }$, affect the statistical comparison between pre- and post-training results a non-linear regression fit of the seven parameter MVC torque function was performed to each Torque vs. Angular velocity ( $T-\omega)$ dataset. This was done first to the individual subject results and subsequently to the combined datasets, with the DIFACT upper bound, $\alpha_{\max }$, set successively at $100 \%, 95 \%$ and $90 \%$. This range was chosen as \%VA has been shown to be as low as around $89 \%$ during slow isokinetic concentric contractions of the quadriceps and increase with increasing angular velocity (Babault et al., 2001, 2002; Paillard et al., 2005). The fits for each subject were statistically compared using the extra-sum-of-squares F-test (Motulsky \& Christopoulos, 2004) to establish whether the training intervention had a statistically significant effect on any of the subjects.

The goodness of fit of the resulting maximal voluntary torque-velocity curves was assessed: The values of the determination coefficient, $\mathrm{R}^{2}$, and RMS difference scores from each fit were calculated for every $\alpha_{\max }$ value. A mixed two-way ANOVA 
was used to assess any differences in the $\mathrm{R}^{2}$ and RMS scores per $\alpha_{\max }$ value both within and between the two testing sessions.

The same process was repeated for fitting the DIFACT function to the \%VA values of both testing sessions. The $\mathrm{R}^{2}$ values obtained from the two fits are indicative of how well the fitted functions reproduce the raw $\mathrm{T}-\omega$ and \%VA- $\omega$ profiles and show whether or not the DIFACT function successfully follows the in vivo voluntary neural activation-angular velocity profiles. The degree that the RMS and $\mathrm{R}^{2}$ values change for different values of $\alpha_{\max }$ is a measure of the robustness of the DIFACT function. In order for the DIFACT function to be robust no significant differences between the results of the fits with the different $\alpha_{\max }$ values should be observed within the pretraining data, or in the post-training data.

The curve fit and statistical analysis was performed using Matlab (The MathWorks Inc., Natick, MA, USA). A statistical level of significance, $p<0.05$, was used throughout.

\section{RESULTS}

Applying the extra-sum-of-squares F-Test on the seven parameter MVC function fit to the torque-angular velocity dataset, for $\alpha_{\max }=100 \%$, showed that 3 out of 6 subjects had a significant $(p<0.05)$ higher torque output post-testing. The same outcome was obtained when the $\alpha_{\max }$ values were set equal to $95 \%$ and $90 \%$.

There was no significant difference between the $R^{2}$ values of the three fits with different $\alpha_{\max }$ values for both pre- and post-training datasets ( $p=0.95 \& p=0.99$ respectively) for any of the six subjects. The mean $R^{2}$ values across all subjects (group mean) were 0.95 for pre-training (range 0.84 to 0.99 ) and 0.96 for posttraining (range 0.89-0.99). Additionally, there was no significant difference $(p \geq 0.05)$ in the group mean $\mathrm{R}^{2}$ scores between sessions. Similarly, there was no significant difference between the RMS scores of the three fits with different $\alpha_{\max }$ values for any of the six subjects (Table 1 ) either pre- or post-training ( $p=0.92 \& 0.96$ respectively). The RMS score variation was much greater between subjects than any variation due to changing $\alpha_{\max }$ within subjects. Group mean RMS scores were 18 (range 7.6-45.7) and 13.9 (range 5.3-26.7) for pre- and post-training sessions respectively. There was no significant difference $(p \geq 0.05)$ in the group mean RMS scores between the two sessions. 
Table 1 RMS differences for the 7-parameter torque function fit to the T- $\omega$ data for $\alpha_{\max }=100 \%, 95 \%, 90 \%$,

\begin{tabular}{|c|c|c|c|c|c|c|}
\hline & \multicolumn{3}{|c|}{ Pre training } & \multicolumn{3}{|c|}{ Post training } \\
\hline & $\begin{array}{l}\alpha_{\max }=100 \\
\%\end{array}$ & $\alpha_{\max }=95 \%$ & $\begin{array}{l}\alpha_{\max }=90 \\
\%\end{array}$ & $\begin{array}{l}\alpha_{\max }=100 \\
\%\end{array}$ & $\alpha_{\max }=95 \%$ & $\begin{array}{l}\alpha_{\max }=90 \\
\%\end{array}$ \\
\hline Subject 1 & 41.2 & 45.7 & 45.6 & 8.8 & 8.5 & 8.5 \\
\hline Subject 2 & 11.2 & 13.8 & 11.3 & 4.7 & 5.3 & 5.3 \\
\hline Subject 3 & 14.5 & 17.2 & 14.4 & 26.6 & 27.2 & 26.7 \\
\hline Subject 4 & 8.0 & 8.0 & 8.0 & 7.0 & 7.0 & 7.0 \\
\hline Subject 5 & 7.6 & 7.7 & 12.1 & 15.1 & 15.1 & 15.1 \\
\hline Subject 6 & 17.5 & 24.4 & 16.0 & 20.5 & 20.5 & 20.5 \\
\hline
\end{tabular}

When the DIFACT function was fitted on the \%VA dataset the $\mathrm{R}^{2}$ values per testing session were not significantly different $(p \geq 0.05)$, however, the post-training group mean $\mathrm{R}^{2}$ score, 0.68 , was significantly $(\mathrm{p}<0.05)$ higher than the pre-training value, 0.57 . The respective ranges were $0.32-0.84$ and $0.32-0.89$. Again the $\mathrm{R}^{2}$ score variation was much greater between subjects than any variation due to changing $\alpha_{\max }$ within subjects. Similarly, there was no significant difference between the RMS scores of the three fits, with different $\alpha_{\max }$ values, to the \%VA- $\omega$ profiles for either pre- or post-training ( $p=0.98 \& 0.63$ respectively). The RMS score variation was greater between subjects than any variation due to changing $\alpha_{\max }$ within subjects. The group mean RMS post-training score, 0.09 (range $0.04-0.15$ ) was significantly ( $p$ $<0.05)$ lower than the respective pre-training mean RMS score $(0.11$, range 0.062 0.182 ). Mean \%VA values across eight different isovelocities showed a general rise from mid 60 s to low 90 s but with post training generally being $5 \%$ higher (Table 2 ). Mean $\alpha_{\min }$ was similar to the lowest $\%$ VA values at around $60 \%$ for pre-training and $64 \%$ for post training (Table 3 )

Table 2 Mean \%VA values and standard deviations (SD) pre- and post-training for each angular velocity (deg/sec).

\begin{tabular}{lcc}
\hline \multirow{2}{*}{$\begin{array}{l}\text { Angular } \\
\text { Velocity }\end{array}$} & \multicolumn{2}{c}{ Mean \%VA \pm SD } \\
\cline { 2 - 3 } & Pre Training & Post Training \\
$\mathbf{- 2 5 0}$ & $67.4 \pm 12.60$ & $68.5 \pm 11.90$ \\
$\mathbf{- 1 5 0}$ & $63.6 \pm 15.60$ & $72.0 \pm 7.50$ \\
$\mathbf{- 1 0 0}$ & $64.8 \pm 20.30$ & $69.7 \pm 11.70$ \\
$\mathbf{- 5 0}$ & $67.1 \pm 10.36$ & $76.1 \pm 4.36$ \\
$\mathbf{0}$ & $86.3 \pm 9.15$ & $89.2 \pm 3.49$ \\
$\mathbf{5 0}$ & $85.5 \pm 5.17$ & $88.7 \pm 3.93$ \\
$\mathbf{1 0 0}$ & $88.6 \pm 7.88$ & $94.1 \pm 2.58$ \\
$\mathbf{1 5 0}$ & $91.3 \pm 4.24$ & $94.7 \pm 1.33$ \\
\hline
\end{tabular}

Table 3. Mean $\alpha_{\min }$ values in $\%$ and SD pre- and post-training for each value of $\alpha_{\max }$

\begin{tabular}{lcc}
\hline $\boldsymbol{\alpha}_{\max (\%)}$ & \multicolumn{3}{c}{ Mean $\boldsymbol{\alpha}_{\min } \pm$ SD } \\
\cline { 2 - 3 } & Pre Training & Post Training \\
$\mathbf{1 0 0}$ & $62 \pm 0.04$ & $67 \pm 0.05$ \\
$\mathbf{9 5}$ & $60 \pm 0.02$ & $64 \pm 0.04$ \\
$\mathbf{9 0}$ & $61 \pm 0.02$ & $63 \pm 0.05$ \\
\hline
\end{tabular}


Figure 1 illustrates how the DIFACT-function obtained from fitting the seven parameter function to the torque-angular velocity dataset of Subjects 2 and 4 follows the raw ITT-angular velocity dataset for the three $\alpha_{\max }$ values and how this compares against the DIFACT-function fit to the ITT-angular velocity dataset.
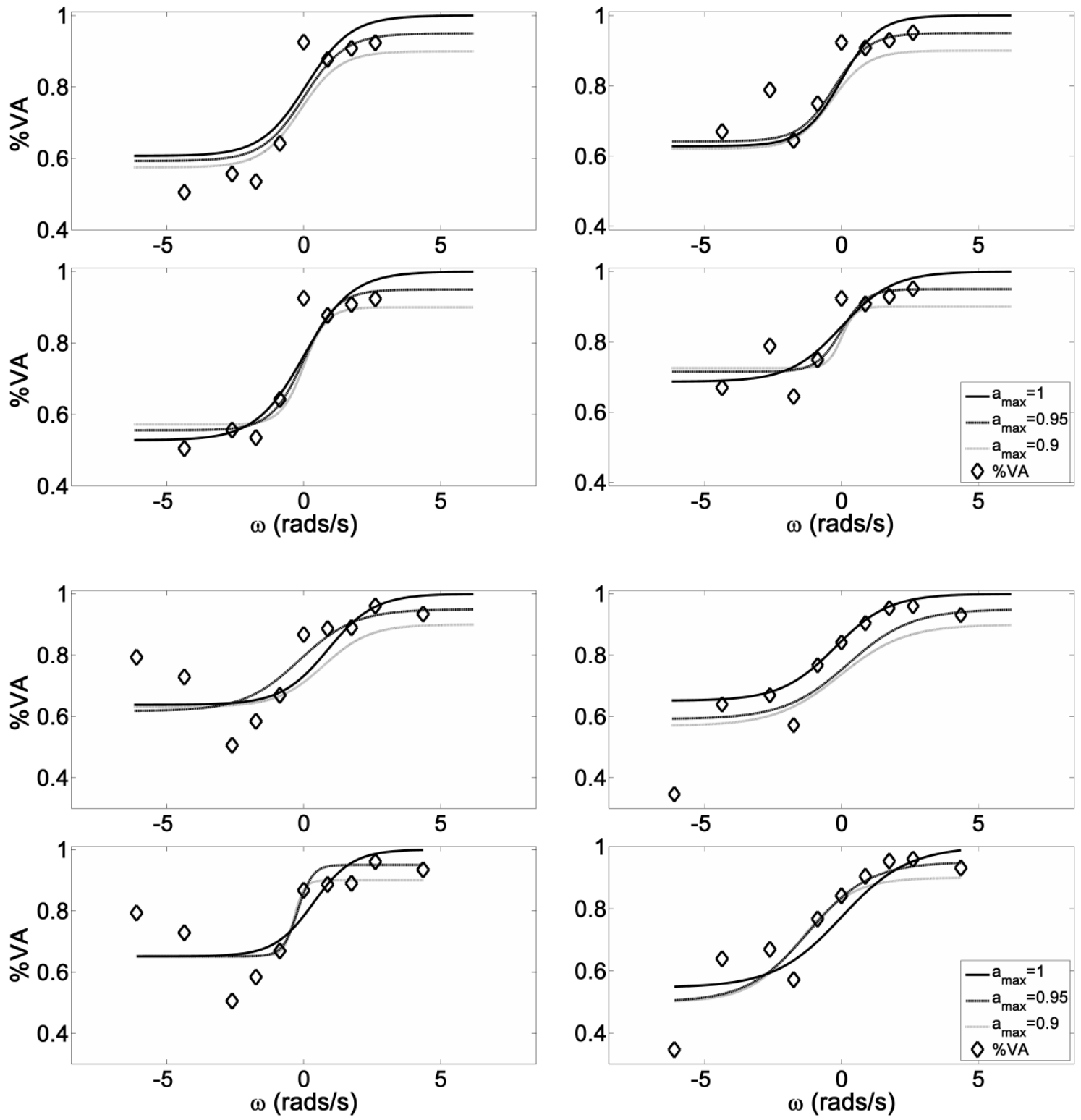

Figure 1 The four graphs show how the DIFACT function compares with the raw $\%$ VA- $\omega$ data set for $\alpha_{\max }=100 \%, 95 \%, 90 \%$ for Subjects 2 (a) and 4 (b). Graphs on the left correspond to the pre-training values and on the right to the post-training ones. The top two graphs, per subject, show the DIFACT function from the seven parameter fit superimposed on the \%VA- $\omega$ data set. In the bottom two graphs the DIFACT function has been fitted to the \%VA- $\omega$ data set directly and again plotted against the respective \%VA- $\omega$ values. 


\section{DISCUSSION}

The aim of this work was to determine how well the three-parameter exponential differential activation function DIFACT (Pain \& Forrester, 2009) reproduces the invivo $\mathrm{T}-\omega$ and $\% \mathrm{VA}-\omega$ profiles and whether changing the value of the maximum activation level, $\alpha_{\max }$, in DIFACT (Pain \& Forrester, 2009) would affect its robustness. Results show that the MVC torque function reproduces the T- $\omega$ raw data set very well irrespective of the $\alpha_{\max }$ value. The DIFACT function is also successful in reproducing the raw \%VA- $\omega$ data set albeit not to the same extent as the torque function. This is, probably, due to the increased variability in the \%VA values of some subjects obtained from eccentric contractions, mainly during the pre-training session. A number of factors may have been the cause of the observed variability such as the expectation of noxious stimuli (Shield \& Zhou, 2004) or a possible variation between trials in the joint angle where the stimulus was applied (Tillin et al. 2012). However, the DIFACT function appears to behave consistently irrespective of the range of \%VA values or the presence of outlier points (Figure 1).

In previous studies (Yeadon et al., 2006; Pain \& Forrester, 2009, Forrester et al., $2011)$, the maximum activation level of DIFACT, $\alpha_{\max }$, was assumed to be equal to $100 \%$, corresponding to full activation, $100 \%$, of the muscles at high concentric velocities. However, activation deficits of 5-30\% have been reported during high concentric contractions (Babault et al., 2001, 2002; Paillard et al., 2005) in agreement with the results of this study showing that the muscles do not attain full activation. Using $\alpha_{\max }$ values between $90 \%$ and $95 \%$ might be a better representation of the maximum activation of muscles during voluntary efforts. In the current study an $\alpha_{\max }$ value of $100 \%$ appears to be the appropriate value to use for the posttraining as four subjects achieved the lowest RMS scores for that value post-training and there are strong indications of increased neuromuscular activation, due to the training protocol (Table 2). For the pre -training datasets an $\alpha_{\max }$ value of $95 \%$ seems to be more suitable as the overall activation is lower, suggesting that setting the $\alpha_{\max }$ at $100 \%$ may be excessive. However, setting $\alpha_{\max }=90 \%$ is likely too low to account for fast concentric contractions where the muscle activation appears to be enhanced compared to other types of contraction (Tillin et al. 2012) and surpassed the values reported here (Table 2). Given this and the robustness of the fitting methods, setting $\alpha_{\max }$ to $100 \%$ in most cases is likely an assumption that will not introduce any meaningful errors. Indeed, when the mean raw torque outputs at $350 \%$ are compared against the mean values of the 7-parameter MVC function fits, at $350 \%$, with $\alpha_{\max }$ set at $100 \%$ and $95 \%$, the differences were $3.7 \%$ and $1.3 \%$ respectively for the pre-training results and $0.9 \%$ and $0.8 \%$ post-training. Moreover, the mean $\alpha_{\min }$ value of $67 \%$ compares very well with the mean $\%$ VA value of $69 \%$ at $-250 \%$ s (a velocity where the inhibition would be significant) post-training suggesting the use of a higher $\alpha_{\max }$ value for analysing post-training datasets. The higher posttraining $\mathrm{R}^{2}$ values imply an improved fit in line with the expectation of a more consistent activation pattern and activation profile, with respect to angular velocities, post-training.

Overall the mean $\mathrm{R}^{2}$ values of the DIFACT fit to the \%VA- $\omega$ pre- and post-training $(0.57 \vee 0.68)$, the agreement between the $\alpha_{\min }$ and $\% \vee A$ values at $-250 \%$, the qualitative agreement between the fitted DIFACT function and the raw datasets observed in Figure 1 suggest that the neural inhibition (Westing, 1988; Dudley et al., 
1990; Weber \& Kriellaars, 1997) may be represented by means of an S-shaped function such as the DIFACT function. Concluding, it has been shown that the exponential DIFACT function remains robust for various values of the maximum level of activation value, $\alpha_{\max }$, and it represents well the neural inhibition of the knee extensors during fast eccentric and slow concentric contractions.

Conflict of Interest Statement

Neither author has any conflict of interests

\section{References}

Babault, N., Pousson, M., Ballay, Y., Van Hoecke, J., 2001. Activation of human quadriceps femoris during isometric, concentric and eccentric contractions. Journal of Applied Physiology 91, 2628-2643.

Babault, N., Pousson, M., Michaut, A., Ballay, Y., Van Hoecke, J., 2002. EMG activity and voluntary activation during knee-extensor concentric torque generation. European Journal of Applied Physiology 86, 541-547.

Dudley, G. A., Harris, R. T., Duvoisin, M. R., Hather, B. M., Buchanan, P., 1990. Effect of voluntary vs. artificial activation on the relationship of muscle torque to speed. Journal of Applied Physiology 69, 2215-2221.

Folland, J. P., Williams, A. G., 2007. Methodological issues with the interpolated twitch technique. Journal of Electromyography and Kinesiology 17, 317-327.

Forrester, S. E., Yeadon, M. R., King, M. A., Pain, M.T.G., 2011. Comparing different approaches for determining joint torque parameters from isovelocity dynamometer measurements. Journal of Biomechanics 44, 955-961.

Lewis, M. G. C., King, M., A., Yeadon, M., R., Conceição, F., 2012. Are joint torque models limited by an assumption of monoarticularity? Journal of Applied Biomechanics 28, 520-529.

Motulski, H., Christopoulos, A., 2004. Fitting Models to Biological Data using Linear and Nonlinear Regression. 1st ed., Oxford University Press.

Paillard, T., Margnes, E., Maitre, J., Chaubet, V., Francois, Y., Gonzalec, G., Borel, L., 2005. Electrical stimulation superimposed onto voluntary muscular contraction. Sports Medicine 35, 951-966.

Pain, M. T. G., Forrester, S., 2009. Predicting maximum eccentric strength from surface EMG measurements. Journal of Biomechanics 42, 1598-1603.

Pain, M. T. G., Young, F., Kim, J., Forrester, S. E., 2013. The torque-velocity relationship in large human muscles: maximum voluntary versus electrically stimulated behaviour. Journal of Biomechanics 46, 645-650.

Tillin, N. A., Pain, M. T. G., Folland, J. P., 2012. Contraction type influences the human ability to use available torque capacity of skeletal muscle during explosive efforts. Proceedings of the Royal Society B 279, 2106-2115.

Shield, A., Zhou, S., 2004. Assessing voluntary muscle activation with the twitch interpolation technique. Sports Medicine 34, 253-267.

Weber, S., Kriellaars, D., 1997. Neuromuscular factors contributing to in vivo eccentric moment generation. Journal of Applied Physiology 83, 40-45.

Westing, S. H., 1988. Eccentric and concentric torque-velocity characteristics of the quadriceps femoris in man. European Journal of Applied Physiology 58, 100-104.

Westing, S. H., Seger, J. Y., \& Thorstensson, A., 1990. Effects of electrical stimulation on eccentric and concentric torque -velocity relationships during knee extension in man. Acta Physiologica Scandinavica, 140, 17-22. 
Westing, S. H., Cresswell, A. G., Thorstensson, A., 1991. Muscle activation during maximal voluntary eccentric and concentric knee extension. European Journal of Applied Physiology 62, 104-108.

Yeadon, M. R., King, M. A., Wilson, C., 2006. Modelling the maximum voluntary joint torque/angular velocity relationship in human movement. Journal of Biomechanics 39, 476-482. 